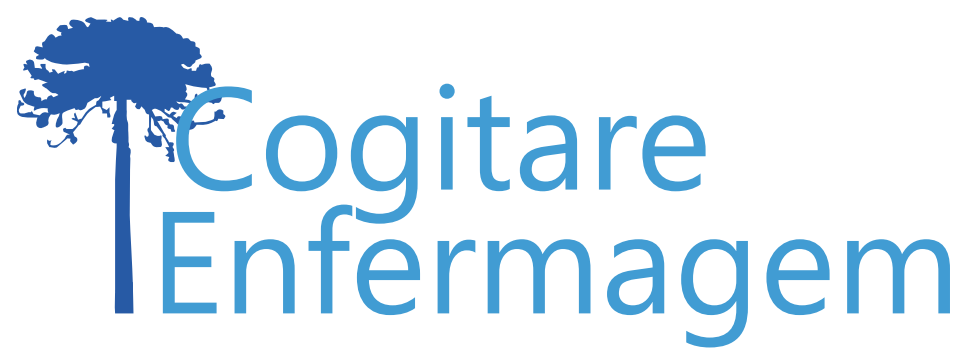

\title{
PREVALÊNCIA DA PROBABILIDADE DE TRANSTORNO MENTAL E FATORES ASSOCIADOS ENTRE INDIVÍDUOS PÓS CIRURGIA BARIÁTRICA*
}

Sara Borges Estevão1, Lauany Martins dos Santos Carneiro², Roselma Lucchese ${ }^{3}$, Ivânia Vera ${ }^{4}$, Valéria Duarte Gregório ${ }^{5}$, Graciele Cristina Silva ${ }^{6}$, Moisés Fernandes Lemos ${ }^{7}$

\section{RESUMO}

Objetivo: estimar a prevalência da probabilidade de transtorno mental comum e fatores associados entre indivíduos submetidos à cirurgia bariátrica.

Métodos: estudo transversal, realizado em 303 indivíduos na região central do Brasil. Aplicou-se instrumento para rastreamento de transtornos mentais e realizou-se análise de regressão logística, com cálculo das razões de prevalência.

Resultados: 50,2\% apresentaram probabilidade de transtorno mental comum. Na análise múltipla, permaneceram associadas ao transtorno mental comum insônia (razão de prevalência: 1,132; IC95\% 1,020-1,256; $p=0,020$ ), compulsão alimentar (razão de prevalência: 1,429; IC95\% 1,2441,$641 ; p=0,000$ ), ter visto ou ouvido coisas que não existiam na abstinência de álcool (razão de prevalência: 1,130; IC95\% 1,033-1,236; $p=0,007$ ), ter sempre bebida alcoólica por perto (razão de prevalência: 1,330; IC95\% 1,182-1,518; $\mathrm{p}=0,000$ ) e ter sido criança/adolescente obeso (razão de prevalência: 0,885; IC95\% 0,786-0,997; $p=0,046)$.

Conclusão: os resultados reforçam a importância da sistematização do cuidado de Enfermagem nas ações de saúde em equipe multidisciplinar.

DESCRITORES: Cirurgia Bariátrica; Transtornos mentais; Enfermagem; Alcoolismo; Obesidade.

*Artigo extraído da dissertação de mestrado "Prevalência do consumo de álcool em pacientes submetidos à cirurgia bariátrica no sudeste goiano". Universidade Federal de Goiás, 2015.

\section{COMO REFERENCIAR ESTE ARTIGO:}

Estevão SB, Carneiro LM dos S, Lucchese R, Vera I, Gregório VD, Silva GC, et al. Prevalência da probabilidade de transtorno mental e fatores associados entre indivíduos pós cirurgia bariátrica. Cogitare enferm. [Internet]. 2020 [acesso em "colocar data de acesso, dia, mês abreviado e ano"]; 25. Disponível em: http://dx.doi.org/10.5380/ ce.v25i0.66846.

${ }^{1}$ Enfermeira. Universidade Federal de Goiás. Catalão, GO, Brasil. 9

${ }^{2}$ Enfermeira. Universidade Federal de Goiás. Catalão, GO, Brasil.

${ }^{3}$ Enfermeira. Doutora em Enfermagem. Docente da Universidade Federal de Goiás. Catalão, GO, Brasil. $\odot$

${ }^{4}$ Enfermeira. Doutora em Enfermagem. Docente da Universidade Federal de Goiás. Catalão, GO, Brasil.

${ }^{5}$ Nutricionista. Mestre em Gestão Organizacional. Docente do Centro Universitário Una. Catalão, GO, Brasil. $\bigcirc$

${ }^{6}$ Nutricionista. Doutora em Ciências da Saúde. Docente da Universidade Federal de Goiás. Catalão, GO, Brasil. (-)

${ }^{7}$ Psicólogo. Pós-doutor em Saúde Coletiva. Docente na Universidade Federal de Goiás. Catalão, GO, Brasil. 


\title{
PREVALENCE OF THE PROBABILITY OF MENTAL DISORDER AND ASSOCIATED FACTORS AMONG INDIVIDUALS AFTER BARIATRIC SURGERY
}

\begin{abstract}
Objective: To estimate the prevalence of the probability of common mental disorder and associated factors among individuals undergoing bariatric surgery.

Methods: A cross-sectional study carried out with 303 individuals in the central region of Brazil. An instrument for tracking mental disorders was applied and logistic regression analysis was performed, calculating the prevalence ratios.

Results: $50.2 \%$ were likely to have common mental disorder. In the multiple analysis, the following remained associated with the common mental disorder: insomnia (prevalence ratio: $1.132 ; 95 \% \mathrm{Cl} 1.020-1.256 ; p=0.020$ ), binge eating (prevalence ratio: $1.429 ; 95 \% \mathrm{Cl}$ $1.244-1.641 ; p=0.000)$, having seen or heard things that did not exist in alcohol abstinence (prevalence ratio: $1.130 ; 95 \% \mathrm{Cl} 1.033-1.236 ; p=0.007)$, always having alcoholic beverages nearby (prevalence ratio: $1.330 ; 95 \% \mathrm{Cl} 1.182-1.518 ; p=0.000)$ and having been an obese child/adolescent (prevalence ratio: $0.885 ; 95 \% \mathrm{Cl} 0.786-0.997 ; p=0.046$ ).

Conclusion: The results reinforce the importance of systematizing Nursing care in health actions in a multidisciplinary team.
\end{abstract}

DESCRIPTORS: Bariatric Surgery; Mental disorders; Nursing; Alcoholism; Obesity.

\section{PREVALENCIA DE LA PROBABILIDAD DE PADECER TRASTORNO MENTAL Y FACTORES ASOCIADOS ENTRE INDIVIDUOS LUEGO DE SER SOMETIDOS A CIRUGÍA BARIÁTRICA}

\section{RESUMEN:}

Objetivo: estimar la prevalencia de la probabilidad de padecer trastorno mental común y factores asociados entre individuos sometidos a cirugía bariátrica.

Métodos: estudio transversal realizado con 303 individuos en la región central de Brasil. Se aplicó un instrumento para el seguimiento de trastornos mentales y se realizó un análisis de regresión logística, con cálculo de las razones de prevalencia.

Resultados: el 50,2\% presentó probabilidad de padecer trastorno mental común. En el análisis múltiple, las siguientes variables permanecieron asociadas al trastorno mental común: insomnio (razón de prevalencia: 1,132; IC95\% 1,020-1,256; $p=0,020$ ), alimentación compulsiva (razón de prevalencia: 1,429; IC95\% 1,244-1,641; $p=0,000)$, haber visto u oído cosas que no existían durante la abstinencia alcohólica (razón de prevalencia: 1,130; IC95\% 1,033-1,236; $p=0,007$ ), tener siempre alguna bebida alcohólica cerca (razón de prevalencia: 1,330; IC95\% 1,1821,$518 ; p=0,000$ ) y haber sido obeso en la niñez y/o en la adolescencia (razón de prevalencia: 0,$885 ;$ IC95\% 0,786-0,997; $p=0,046$ ).

Conclusión: los resultados refuerzan la importancia de sistematizar los cuidados de Enfermería en las acciones de atención a la salud del equipo multidisciplinar.

DESCRIPTORES: Cirugía bariátrica; Trastornos mentales; Enfermería; Alcoholismo; Obesidad. 
O transtorno mental comum (TMC) representa aproximadamente $12 \%$ do total de doenças mentais no mundo e constitui agravo frequente na população mundial e brasileira, abrangendo um terço da população nas diversas faixas etárias ${ }^{(1,2)}$. Caracterizase por sintomas depressivos, de ansiedade, insônia, irritabilidade, fadiga, dificuldade de concentração, esquecimento e queixas somáticas ${ }^{(3)}$.

Diversos outros agravos crônicos à saúde associam-se ao TMC; dentre estes, estudos recentes postularam a associação entre a obesidade e sintomas de transtornos mentais ${ }^{(4-6)}$. A obesidade é uma doença nutricional considerada um problema de saúde pública, e a Cirurgia Bariátrica (CB), por sua vez, tem sido considerada a forma de tratamento de maior eficácia (7), atribuindo-se o sucesso deste procedimento à perda de peso ponderal definitiva e permanente, sendo a técnica mais comum bypass gástrico em $Y$ de Roux (Fobi-Capella). Contudo, tem-se verificado o desenvolvimento de transtornos alimentares e psiquiátricos, em sua maioria decorrentes da frustração da expectativa em torno da perda ponderal almejada, isto é, quando não são atingidos os objetivos do paciente pós $\mathrm{CB}^{(4,5)}$.

Apesar da literatura ter postulado a relação entre obesidade e sintomas de transtornos mentais, sobretudo ansiedade e depressão( ${ }^{(8,9)}$, as investigações se estes transtornos e sintomas permanecem ou cessam após a realização do procedimento cirúrgico em indivíduos ainda são escassas ${ }^{(6)}$, evidenciando uma lacuna de conhecimento.

Estudo realizado entre indivíduos usuários da Atenção Primária em Saúde (APS) da região central do Brasil detectou a prevalência de 31,47\% de probabilidade para o desenvolvimento de TMC antes da CB. Neste ínterim, a obesidade atinge aproximadamente $29,4 \%$ da população atendida na APS ${ }^{(2)}$. Diante dos altos índices de TMC e obesidade apresentados em estudo prévio realizado com outras populações, faz-se importante rastrear o TMC em indivíduos pós-CB bem como os desfechos pós-operatórios.

Além disso, outros agravos também têm sido considerados fatores de risco para o desenvolvimento de TMC, como tabaco, consumo indevido de álcool e alterações no padrão de sono. Estudos recentes verificaram a associação entre o consumo de álcool em pacientes pós $C B$, com prevalência de tempo de pós-operatório entre seis meses a três anos, maioria de sexo masculino e prevalência de $16,5 \%$ de consumo de tabaco nos últimos 30 dias $^{(9,10)}$. Em relação às alterações no padrão de sono, a ocorrência destes distúrbios em indivíduos com transtornos mentais pós CB são escassas. As discretas investigações realizadas encontraram presença de apneia do sono, com consequente privação do sono, sonolência diurna e alteração no metabolismo em indivíduo candidatos a $\mathrm{CB}^{(11)}$ com taxa de remissão de $60 \%$ pós $\mathrm{C}^{\mathrm{B}^{(12)}}$.

Esta investigação objetiva estimar a prevalência da probabilidade de TMC e de fatores associados entre indivíduos submetidos à CB.

\section{MÉTODO}

Estudo transversal realizado em um município de médio porte, de relevante importância econômica e social na região central do Brasil e referência no âmbito da atenção primária à saúde da comunidade.

A amostra do estudo compôs-se de indivíduos pós CB que realizaram o procedimento pelas técnicas de bypass gástricos em $Y$ de Roux, sleeve e gastrectomia parcial com atendimento no ambulatório para pacientes de recursos suplementares (convênios) e particulares no período mínimo de 60 dias pós $C B$ com idade $\geq 18$ anos. Excluíramse aqueles que se apresentavam em período gestacional, com diagnóstico de doença 
mental grave avaliado pelo questionário Self-Reporting Questionnaire (SRQ-20) e doenças carcinogênicas. A escolha desta população específica pelos sistemas suplementar e particular decorre da crescente evolução do número de procedimentos cirúrgicos no Brasil, além de atualmente ser uma lacuna de conhecimento, uma vez que a maioria dos estudos são realizados por serviços públicos de saúde. Estima-se um crescimento exponencial do número de cirurgias pelo sistema suplementar dez vezes maior do que as realizadas pelo Sistema Único de Saúde (SUS) ${ }^{(13)}$.

A amostra foi calculada com poder estatístico de $80 \%(\beta=20 \%)$, grau de significância de $5 \%(a=0,05)$, efeito de desenho de 3,0 e frequência antecipada de uso problemático de álcool de $6,5 \%$ em população pós $\mathrm{CB}^{(14)}$. Considerou-se acréscimo de $10 \%$ para possíveis perdas amostrais, resultando em um $\mathrm{N}=309$ indivíduos. A amostragem deu-se por conveniência.

Os indivíduos foram recrutados por contato telefônico, entre março de 2015 e março de 2016. Aplicou-se face a face em ambiente privativo instrumento construído por profissionais expertises na área, previamente validado, contendo dados sociodemográficos, situação de saúde, histórico familiar, hábitos de vida e condição após CB. Para rastrear os possíveis casos de TMC, utilizou-se o SRQ-20 na versão validada para a população brasileira, composto por 20 questões dicotômicas (sim equivale a um ponto; não corresponde a zero ponto), com resultado de escore $\geq 7$ para probabilidade de TMC ${ }^{(15)}$. Definiu-se como variável dependente do estudo o escore do SRQ-20 dicotomizado em apresentar probabilidade para TMC (não vs. sim) e as variáveis independentes relacionadas no Quadro 1.

Quadro 1 - Variáveis abordadas e possibilidades de resposta no questionário aplicado. Brasil Central, BR, 2015-2016 (continua)

\begin{tabular}{|c|c|c|}
\hline $\mathbf{N}$ & Variáveis & Possibilidade de respostas \\
\hline 1 & Sexo & feminino vs. masculino \\
\hline 2 & Renda & $\begin{array}{l}>\mathrm{R} \$ 1.581,00 \text { vs. } \\
\leq \mathrm{R} \$ 1.581,00\end{array}$ \\
\hline 3 & Estado civil & $\begin{array}{l}\text { vive com companheiro vs. } \\
\text { vive sem companheiro }\end{array}$ \\
\hline 4 & Possuir filhos & não vs. sim \\
\hline 5 & Ter religião & não vs. sim \\
\hline 6 & Apresentar insônia & não vs. sim \\
\hline 7 & $\begin{array}{l}\text { Diagnóstico de depressão - já ter sido diagnosticado por um médico } \\
\text { ou ter feito tratamento para depressão }\end{array}$ & não vs. sim \\
\hline 8 & $\begin{array}{l}\text { Diagnóstico de ansiedade - já ter sido diagnosticado por um médico } \\
\text { ou ter feito tratamento para ansiedade }\end{array}$ & não vs. sim \\
\hline 9 & Atividade física $\geq 3$ vezes na semana & não vs. sim \\
\hline 10 & $\begin{array}{l}\text { Deficiência de vitamina B12 - já ter recebido resultado de exames } \\
\text { com déficit de vitamina B12 pós CB }\end{array}$ & não vs. sim \\
\hline 11 & $\begin{array}{l}\text { Deficiência de vitamina } D \text { - já ter recebido resultado de exames com } \\
\text { deficiência de vitamina } D \text { pós } C B \text { (valores abaixo dos parâmetros } \\
\text { bioquímicos de vitamina } D<10 \text { ng/Ml foram considerados como } \\
\text { diagnóstico de deficiência) }{ }^{(7)}\end{array}$ & não vs. sim \\
\hline 12 & $\begin{array}{l}\text { Deficiência de ferro - já ter recebido resultado de exames com } \\
\text { deficiência de ferro pós CB (valores abaixo dos parâmetros } \\
\text { bioquímicos de ferro sérico }<60 \mu \mathrm{g} / \mathrm{Dl} \text { foram considerados como } \\
\text { diagnóstico de deficiência) }{ }^{(7)}\end{array}$ & não vs. sim \\
\hline
\end{tabular}




\begin{tabular}{|l|l|l|}
\hline 13 & $\begin{array}{l}\text { Compulsão alimentar após CB, compreendendo compulsão alimentar } \\
\text { como consumo excessivo de alimentos em um curto período de } \\
\text { tempo pós CB }\end{array}$ & não vs. sim \\
\hline 14 & Ter sido criança ou adolescente obeso & não vs. sim \\
\hline 15 & Ter familiar obeso & não vs. sim \\
\hline 16 & Ter familiar que tentou suicídio & não vs. sim \\
\hline 17 & $\begin{array}{l}\text { Beber diariamente - no mínimo uma dose de bebida alcoólica por } \\
\text { dia }\end{array}$ & não vs. sim \\
\hline 18 & Abstinência de álcool & não vs. sim \\
\hline 19 & Ter visto ou ouvido coisas que não existiam & não vs. sim \\
\hline 20 & Tentar parar de beber e não conseguir & não vs. sim \\
\hline 21 & Ter sempre bebida alcoólica por perto & não vs. sim \\
\hline
\end{tabular}

Os dados foram digitados em dupla conferência e analisados no software Statistics/ Data Analysis (Stata), versão 14.0. Para as respostas do instrumento SRQ-20, foi realizado o teste de confiabilidade alfa de Cronbach.

As variáveis categóricas foram expressas em frequências absolutas e relativas, prevalência e intervalo de confiança de 95\% (IC 95\%). As contínuas em média e Desvio Padrão (DP). As análises estatísticas bivariada e múltipla foram obtidas pela Razão de Prevalência (RP) com medida de efeito de poisson e IC $95 \%$. As variáveis com $p<0,10$ foram selecionadas para o modelo múltiplo. $\mathrm{Na}$ análise múltipla, consideraram-se associadas as variáveis com $\mathrm{p}<0,05$.

Este manuscrito faz parte de um projeto matriz intitulado "Cuidado em Saúde Mental nos Serviços de Saúde do Sudeste", que analisou a atenção em saúde mental no município, aprovado pelo Comitê de Ética em Pesquisa da Universidade Federal de Goiás, parecer $523.834 / 2014$.

\section{RESULTADOS}

A amostra do estudo foi composta por 317 indivíduos pós $C B$, subtraindo-se 14 perdas por inconsistência dos dados, totalizando 303 indivíduos. Houve prevalência do sexo feminino, com 76,2\% ( $n=231$; IC95\% 71,0-80,9), com média de idade de 37,2 anos (IC95\%36,1-38,3; DP $\pm 9,78)$, etnia/cor da pele branca 67,7\% ( $n=205 ;$ IC95\%62,0-73,3), seguida por pardo/moreno/mulato $26,4 \%$ ( $n=80 ;$ IC $95 \%$ 21,5-31,4); 47,9\% ( $n=145 ;$ IC $95 \%$ $46,5-58,1)$ tinham escolaridade superior a 8 anos de estudo, renda média de $\mathrm{R} \$ 3.625,29$ (IC95\% 19,7-25,3; DP $\pm 24,8$ ) e tempo cirúrgico, em média, de 22,5 meses (IC95\% 19,725,3; DP $\pm 24,8)$.

Dos 303 indivíduos, 50,2\% apresentaram escore de prevalência de probabilidade para TMC, segundo o SRQ-20. Para verificação da confiabilidade das respostas ao instrumento, o alfa de Cronbach foi 0,822 . Na Tabela 1, apresentam-se os fatores associados à prevalência de probabilidade de TMC em análise bivariada e múltipla. 
Tabela 1 - Análise bivariada e múltipla de probabilidade de transtorno mental comum entre indivíduos pós cirurgia bariátrica, segundo o Self-Reporting Questionnaire. Brasil Central, BR, 2015-2016 (continua)

TMC

RP bruta

RP ajustada

Variável

\begin{tabular}{|c|}
\hline $\begin{array}{l}\text { n/total* } \\
N=303\end{array}$ \\
\hline
\end{tabular}

Sexo

\begin{tabular}{lcccc} 
Masculino & $30 / 151$ & 19.7 & $\begin{array}{c}1 \\
1,078\end{array}$ & 0,121 \\
\cline { 1 - 2 } Feminino & $122 / 152$ & 80,3 & $(0,984-1,181)$ & \\
\hline
\end{tabular}

Renda, R\$

\begin{tabular}{ccccc}
\hline$>1,581$ & $29 / 53$ & 54,7 & 1 & 0,802 \\
\cline { 1 - 2 }$\leq 1,581$ & $123 / 250$ & 49,2 & $(0,465-1,498)$ & 0,466 \\
\hline
\end{tabular}

Estado civil

\begin{tabular}{lcccc} 
Com companheiro & $43 / 88$ & 48,8 & $\begin{array}{c}1 \\
1,009\end{array}$ & 0,829 \\
\cline { 1 - 3 } Sem companheiro & $107 / 215$ & 49,7 & $(0,929-1,095)$ & \\
\cline { 1 - 3 } Possui filhos & & & & \\
\cline { 1 - 2 } Não & $39 / 86$ & 45,3 & $\begin{array}{c}1 \\
1,046\end{array}$ & 0,295 \\
\cline { 1 - 3 } Sim & $113 / 217$ & 52,1 & $(0,961-1,138)$ & \\
\hline
\end{tabular}

Tem religião

\begin{tabular}{lcccc}
\hline Não & $11-17$ & 64,7 & 1 & 0,08 \\
\cline { 1 - 2 } $\operatorname{Sim}$ & $141 / 286$ & 49,3 & $(0,955-1,273)$ & 0,18 \\
\hline
\end{tabular}

Apresenta insônia

\begin{tabular}{lllcccc}
\hline Não & $57 / 137$ & 41,6 & 1 & 1 & \\
\cline { 1 - 2 } Sim & $95 / 166$ & 57,2 & $(1,029-1,197)$ & 0,007 & 1,132 & $\mathbf{0 , 0 2 0}$ \\
\hline
\end{tabular}

Atividade física $\geq 3$ vezes/semana

\begin{tabular}{ccccccc}
\hline $\operatorname{Sim}$ & $41 / 107$ & 38,3 & 1 & & 1 & \\
\cline { 1 - 2 } Não & $111 / 196$ & 56,6 & $(1,045-1,293)$ & 0,002 & 1,041 & 0,458 \\
\hline
\end{tabular}

Deficiência de vitamina B12

\begin{tabular}{|c|c|c|c|c|c|c|}
\hline Não & $85 / 189$ & 45,2 & 1 & & 1 & \\
\hline Sim & $67 / 115$ & 58,2 & $\begin{array}{c}1,084 \\
(1,010-1,175)\end{array}$ & $0,0<5$ & $\begin{array}{c}1,038 \\
(0,936-1,150)\end{array}$ & $0,4 / 0$ \\
\hline
\end{tabular}

Deficiência de vitamina $D$

\begin{tabular}{|c|c|c|c|c|c|c|}
\hline Não & $103 / 219$ & 47 & \multirow{2}{*}{$\begin{array}{c}1 \\
1,076 \\
(0,9935-1,167) \\
\end{array}$} & \multirow{2}{*}{0,071} & \multirow{2}{*}{$\begin{array}{c}1 \\
1,019 \\
(0,929-1,118) \\
\end{array}$} & \multirow{2}{*}{0,687} \\
\hline Sim & $49 / 84$ & 58,3 & & & & \\
\hline \multicolumn{7}{|c|}{ Deficiência de ferro } \\
\hline Não & $96 / 217$ & 44,2 & \multirow{2}{*}{$\begin{array}{c}1 \\
1,144 \\
(1,060-1,235) \\
\end{array}$} & \multirow{2}{*}{0,001} & 1 & \multirow{2}{*}{0,123} \\
\hline Sim & $56 / 86$ & 65,1 & & & $(0,978-1,197)$ & \\
\hline \multicolumn{7}{|c|}{ Compulsivo alimentar após CB? } \\
\hline Não & $149 / 298$ & 49,6 & 1 & & 1 & \\
\hline
\end{tabular}




\begin{tabular}{llrrrrr}
\hline $\operatorname{Sim}$ & $4-4$ & 100 & $\begin{array}{c}1,336 \\
(1,286-1,388)\end{array}$ & 0 & $\begin{array}{c}1,429 \\
(1,244-1,641)\end{array}$ & $\mathbf{0 , 0 0 0}$ \\
\hline
\end{tabular}

Foi criança/adolescente obeso?

\begin{tabular}{ccccccc}
\hline Não & $119 / 215$ & 55,3 & 1 & 1 & 0 \\
\cline { 1 - 2 } $\operatorname{Sim}$ & $33 / 88$ & 37,5 & $(0,284-0,803)$ & 0,005 & 0,885 & $\mathbf{0 , 0 4 6}$ \\
\hline
\end{tabular}

Tem familiar obeso?

\begin{tabular}{ccccccc}
\hline Não & $37 / 51$ & 72,5 & 1 & 1 & 0 \\
\cline { 1 - 2 } Sim & $115 / 252$ & 45,6 & $(0,145-0,555)$ & & $(0,808-1,009)$ & 0,073 \\
\hline Familiar tentou suicídio? & & & &
\end{tabular}

Familiar tentou suicídio?

\begin{tabular}{|c|c|c|c|c|c|c|}
\hline Não & $25 / 151$ & 16,6 & \multirow{2}{*}{$\begin{array}{c}1 \\
2,698 \\
(1,638-4,970)\end{array}$} & \multirow{2}{*}{0} & \multirow{2}{*}{$\begin{array}{c}1 \\
1,052 \\
(0,944-1,172)\end{array}$} & \multirow{2}{*}{0,353} \\
\hline Sim & $53 / 152$ & 34,9 & & & & \\
\hline \multicolumn{7}{|c|}{ Bebe diariamente? } \\
\hline Não & $146 / 296$ & 49,3 & \multirow{2}{*}{$\begin{array}{c}1 \\
1,243 \\
(1,075-1,437)\end{array}$} & \multirow{2}{*}{0,003} & 1 & \multirow{2}{*}{0,701} \\
\hline Sim & $6-7$ & 85,7 & & & $(0,722-1,244)$ & \\
\hline
\end{tabular}

Na abstinência de álcool, você viu ou ouviu coisas que não existiam?

\begin{tabular}{|c|c|c|c|c|c|c|}
\hline Não & $133 / 281$ & 47,3 & \multirow{2}{*}{$\begin{array}{c}1 \\
7,048 \\
(2,680-26,622) \\
\end{array}$} & \multirow{2}{*}{0} & \multirow{2}{*}{$\begin{array}{c}1 \\
1,13 \\
(1,033-1,236) \\
\end{array}$} & \multirow{2}{*}{0,007} \\
\hline Sim & $19 / 22$ & 86,4 & & & & \\
\hline \multicolumn{7}{|c|}{ Tentou parar de beber e não conseguiu? } \\
\hline Não & $0 / 151$ & 0 & \multirow{2}{*}{$\begin{array}{c}1 \\
4,263 \\
(1,869-21,127)\end{array}$} & \multirow{2}{*}{0,008} & 1 & \multirow{2}{*}{0,062} \\
\hline Sim & $7 / 152$ & 4,6 & & & $(0,731-1,007)$ & \\
\hline
\end{tabular}

Tem sempre bebida alcoólica por perto?

\begin{tabular}{|c|c|c|c|c|c|c|}
\hline Não & $57 / 131$ & 43,5 & 1 & & 1 & \\
\hline Sim & $15 / 15$ & 100 & $(1,313-1,478)$ & & $(1,182-1,518)$ & \\
\hline
\end{tabular}

Legenda: Em negrito, os números com $\mathrm{p}$-valor $\leq 0,05$. * A variação do " $n$ " analisado para cada variável se deve à recusa de resposta de alguns participantes. Modelo: apresenta insônia, diagnóstico de depressão, diagnóstico de ansiedade, atividade física $\geq 3$ vezes/semana, deficiência de vitamina B12, deficiência de vitamina D, deficiência de ferro, compulsivo alimentar após CB, ter sido criança/adolescente obeso, tem familiar obeso, familiar tentou suicídio, bebe diariamente, na abstinência de álcool você viu ou ouviu coisas que não existem, tentou parar de beber e não conseguiu, tem sempre bebida alcoólica por perto. Controlaram-se as variáveis: deficiência de vitamina B12 e deficiência de ferro, por apresentarem-se confundidoras com apresentar insônia e ter sido criança/adolescente obeso. RP: razão de prevalência; IC95\%: intervalo de confiança de 95\%; CB: cirurgia bariátrica.

Após a análise múltipla, permaneceram associadas positivamente prevalência de probabilidade de TMC com as variáveis apresenta insônia (RP: 1,132; IC95\% 1,020-1,256; $p=0,020)$, compulsão alimentar após CB (RP: 1,$492 ; I C 1,244-1,641 ; p=0,000)$, na abstinência de álcool (RP: 1,130; IC95\% 1,033-1,236; $p=0,007)$ e tem sempre bebida alcoólica por perto (RP: 1,$339 ;$ IC95\% 1,182-1,518; $p=0,000$ ). Associou-se negativamente ao TMC ter sido criança/adolescente obeso (RP: 0,885; IC95\% 0,786-0,997; p=0,046).

Na Figura 1, observa-se a frequência das respostas sim para as 20 questões do instrumento SRQ-20. As questões em que predominaram respostas positivas foram: "Teve sensações desagradáveis no estômago?"; "Sentiu-se nervoso, tenso ou preocupado?", "Cansa-se com facilidade?" e "Dormiu mal?". 


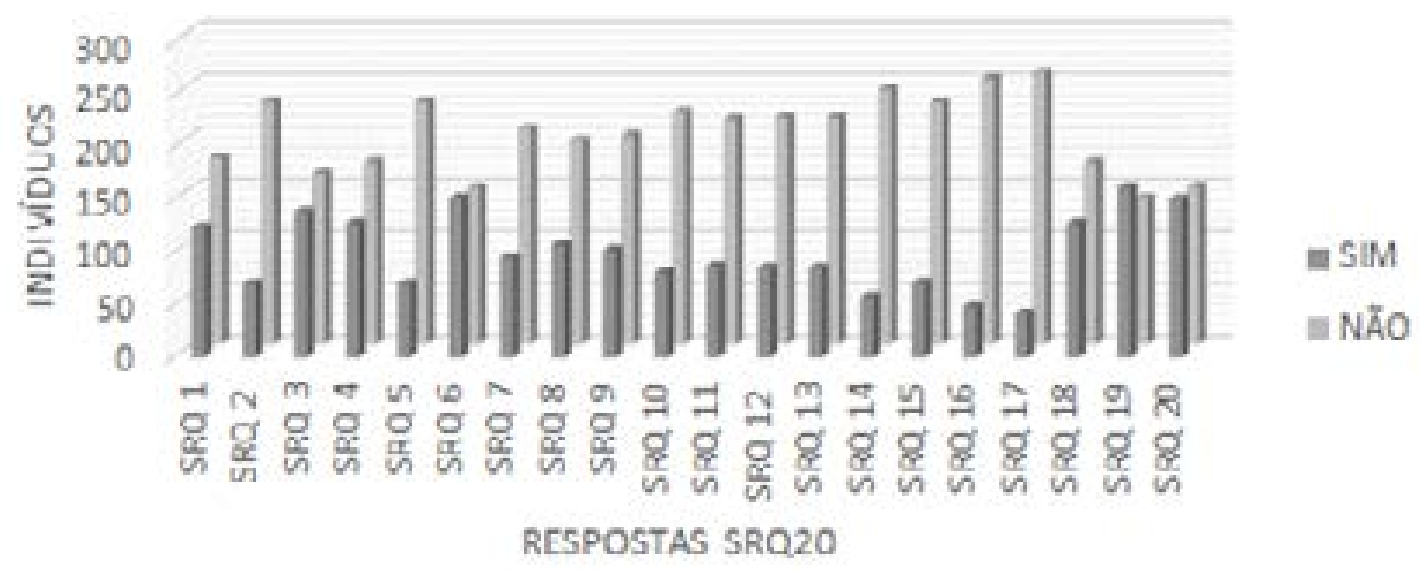

Figura 1 - Respostas sim às 20 questões do Self-Reporting Questionnaire (SRQ-20). Catalão, GO, Brasil, 2015-2016

Legenda: SRQ1: teve dores de cabeça frequente?; SRQ2: perdeu o apetite?; SRQ3: dormiu mal?; SRQ4: assustou-se com facilidade?; SRQ5: teve tremores nas mãos?; SRQ6: se sentiu nervoso, tenso ou preocupado?; SRQ7: teve má digestão?; SRQ8: teve dificuldade para pensar com clareza?; SRQ9: tem se sentido triste ultimamente?; SRQ10: tem chorado mais do que de costume?; SRQ11: teve dificuldade para realizar com satisfação alguma atividade do dia a dia?; SRQ12: teve dificuldade para tomar decisões?; SRQ13: teve dificuldade no serviço?; SRQ14: ficou incapaz de desempenhar um papel útil em sua vida?; SRQ15: perdeu o interesse pelas coisas?; SRQ16: sente-se uma pessoa inútil sem préstimo?; SRQ17: teve ideia de acabar com a própria vida?; SRQ18: você se sente cansado o tempo todo?; SRQ19: teve sensações desagradáveis no estômago?; SRQ20: cansa-se com facilidade?

\section{DISCUSSÃO}

O presente estudo avaliou os fatores associados à prevalência de probabilidade para TMC em pacientes pós CB pelos sistemas suplementar (convênio) e particular, uma vez que este estudo identificou transtornos mentais na população que apresentava obesidade, e, mesmo após a $C B$, a prevalência de sintomas que se enquadram no escopo dos TMC persistiu. Este fato corrobora as práticas de atenção à saúde dessa população, envolvendo a equipe multidisciplinar e ações de enfermagem, como o planejamento da assistência, direcionadas à vulnerabilidade para morbidade psíquica nos indivíduos pós $\mathrm{CB}^{(2)}$.

Além disso, este estudo inova ao investigar uma população sobre a qual atualmente detecta-se lacuna de produção científica. Verifica-se crescente demanda por este procedimento cirúrgico da população proveniente de convênios médicos ${ }^{(13)}$, uma vez que majoritariamente o maior número de pesquisas com CB são realizadas junto ao público vinculado ao SUS, recrutado a partir de hospitais universitários ${ }^{(15-19)}$.

Os achados do presente estudo revelaram alta prevalência de probabilidade de TMC $(50,2 \%)$ quando comparados a estudos brasileiros com população de APS, com valores que variaram entre $20,2^{(2)}$ a $31,4 \%{ }^{(17)}$. Por outro lado, pesquisa realizada em Taiwan com 841 indivíduos pré bariátricos elegíveis para CB encontrou prevalência de TMC de $54,1 \%{ }^{(19)}$. Neste sentido, as altas prevalências de TMC encontradas tanto nos indivíduos pré-CB $(54,1 \%)$ quanto nos indivíduos pós CB $(50,2 \%)$ permitem afirmar que a CB parece não garantir um controle dos sintomas dentre os grupos de $\mathrm{TMC}^{(7)}$.

Outros agravos à saúde decorrentes do TMC que podem afetar a qualidade de vida do indivíduo pós CB foram encontrados neste estudo. A insônia associou-se positivamente $(p=0,020)$ a indivíduos com suspeita de TMC, o que corroborou com estudo prévio que relacionou a obesidade com queixas de insuficiência de sono e insônia ${ }^{(20)}$. Mesmo após a CB, os pacientes apresentaram elevada prevalência de insônia e a duração do sono apresentou associação aos sinais caracterizadores do transtorno(19). Além disso, a curta duração do 
sono (<6 horas/dia) aumenta as chances do desenvolvimento de TMC comparada ao maior tempo de sono (>6 horas/dia)(16). Alterações no padrão de sono podem diminuir as concentrações do hormônio leptina e maiores concentrações do hormônio grelina(21), indicando, por sua vez, uma alteração de perfil endócrino, sendo estimulador de maior fome e menor saciedade ${ }^{(20)}$.

Em relação ao perfil alimentar, o indivíduo que manteve o comportamento alimentar compulsivo após CB $(p=0,000)$ associou-se ao TMC. A compulsão alimentar é um transtorno que pode se vincular aos transtornos psiquiátricos, como ansiedade e depressão, comumente incidentes na população obesa e pós $\mathrm{CB}^{(18)}$. A maior prevalência encontrada deste transtorno reforça a vulnerabilidade desta população na esfera psíquica, em que o indivíduo é afetado pelas mudanças do comportamento alimentar, ressignificando o alimento como amortecedor emocional, ou seja, uma espécie de alívio, o que tende a elevar o risco de ganho ponderal no indivíduo obeso e o reganho durante o pós $\mathrm{CB}^{(22)}$.

Além disso, alterações no funcionamento do sistema nervoso são frequentes nesta população, indicando que a diminuição da ingestão alimentar pode reduzir o tônus serotoninérgico central e predispor o indivíduo à compulsão alimentar. A diminuição da ingestão alimentar e as características como rigidez de pensamento, impulsividade e dificuldade na elaboração das emoções poderiam estar associadas à manifestação do comportamento compulsivo pós $\mathrm{CB}^{(23)^{3}}$.

Por outro lado, nos resultados deste estudo, ter sido criança ou adolescente obeso $(p=0,046)$ esteve negativamente associado com TMC. Os achados são inovadores e divergem de outros estudos, que apontam que a insatisfação com a própria imagem, o risco aumentado de vitimização, transtorno alimentar associado ao bullying e os desfechos de sintomas depressivos relacionados à ansiedade, solidão, isolamento social e problemas comportamentais como internalização, estresse e evasão escolar, realizados em população obesa, associam-se ao risco aumentado de suicídio(24-26). Entretanto, a história pregressa de obesidade na infância protegeria de TMC o adulto obeso, que pode ter recebido tratamento e obtido melhorias significativas nas medidas fisiológicas, comportamentos de saúde e resultados psicossociais ${ }^{(27)}$.

Observou-se ainda que aqueles que relataram possuir bebida alcoólica por perto $(p=0,000)$ apresentaram 33,9\% maior chance de TMC em relação aos que não apresentavam. Em concordância com os achados desta investigação, a literatura documenta que indivíduos pós CB apresentam suscetibilidade à dependência do álcool. Uma das justificativas é o comportamento de impulsividade, que faz o indivíduo se tornar vulnerável à transferência da compulsão alimentar para o abuso de álcool(28). Este comportamento tem sido frequente entre pessoas com dificuldade de tratamentos de controle ponderal, especialmente a impulsividade detectada por transtornos alimentares.

Além disso, a literatura aponta que o consumo de álcool pode atuar na mobilização de sofrimento, proporcionando prejuízo funcional das relações sociais, e a ingestão alcóolica alivia rapidamente o sentimento de aflição ${ }^{(28)}$. Isto, em parte, justificou a necessidade de manter a bebida por perto, o que, neste estudo, associou-se ao TMC.

Os agravos relacionados à maior sensibilidade ao álcool são verificados nos indivíduos pós CB, proporcionando alterações metabólicas, levando-os a um maior risco de intoxicação alcoólica e vulnerabilidade, potencializando os efeitos nocivos, como o descontrole do consumo alcoólico e a intoxicação pelo etanol, relacionado aos níveis séricos deste ${ }^{(21)}$. Pacientes que fazem o uso de álcool após a CB tendem a levar um tempo maior para voltar à sobriedade. Ademais, o uso excessivo de álcool poderia atuar como forma de substituição da compulsão alimentar pós-cirurgia em indivíduos com história de obesidade ${ }^{(28,29)}$.

As variáveis tentar parar de beber, ter sempre bebida por perto e apresentar alterações senso-perceptivas na abstinência carecem de maiores investigações, pois configuram sintomas e comportamentos que reforçam transtornos relacionados ao abuso de álcool, o que tende a elevar o consumo de álcool, induzindo a pessoa a ter sempre a 
bebida por perto. Nos casos de transtorno ocasionados do abuso de álcool, a abstinência representa forma de enfrentar sensações desagradáveis e, na busca de alívio, o indivíduo reforça o padrão de consumo recorrente e intenso ${ }^{(30)}$.

Como limitações deste estudo, pode-se citar o tipo da amostra com indivíduos que passaram pela $C B$, por meio de convênios médicos e recursos próprios, e a associação negativa entre história pregressa de obesidade e TMC, fato sugestivo para a realização de outras investigações mais robustas, com métodos longitudinais, que avaliem a relação entre causalidade e efeito.

\section{CONCLUSÃO}

Dada a expressiva prevalência de probabilidade de transtornos mentais comuns estimados neste estudo e os fatores associados, como insônia, compulsão alimentar, história pregressa de obesidade e abuso de álcool, verificou-se a importância do desenvolvimento de ações voltadas à prevenção do adoecimento mental nesta população.

Ressalta-se a importância da intervenção sistemática da equipe de saúde multidisciplinar e, em especial, o profissional de enfermagem, desde o planejamento da assistência pré-cirúrgica até o monitoramento pós $\mathrm{CB}$. As ações de promoção da saúde devem primar pelo rastreio do transtorno mental comum, sobretudo nos dois anos de seguimento pós-operatório. Estes aspectos visam contribuir para o aperfeiçoamento e a padronização da avaliação, que fornecerão uma melhor atenção dos efeitos a curto e longo prazo da CB.

Os instrumentos utilizados são de fácil manuseio e interpretação, baixo custo e empregabilidade, podendo ser amplamente utilizados, preenchendo uma lacuna encontrada no cuidado do paciente pós CB, além de proporcionar alternativas de cuidado com o paciente como intervenção de enfermagem. Estudos posteriores devem avaliar o impacto da cirurgia sobre o transtorno mental comum durante a evolução da gastroplastia.

\section{REFERÊNCIAS}

1. Knudsen AK, Harvey SB, Mykletun A, Øverland S. Common mental disorders and long-term sickness absence in a general working population. The Hordaland Health Study. Acta psychiatr. scand. [Internet]. 2012 [acesso em 07 jan 2019]; 127(4). Disponível em: http://dx.doi.org/10.1111/j.16000447.2012.01902.x.

2. Lucchese R, Sousa K de, Bonfin S do P, Vera I, Santana FR. Prevalência de transtorno mental comum na atenção primária. Acta paul. enferm. [Internet]. 2014 [acesso em 01 fev 2019]; 27(3). Disponível em: http://dx.doi.org/10.1590/1982-0194201400035.

3. Skapinakis P, Bellos S, Koupidis S, Grammatikopoulos I, Theodorakis PN, Mavreas V. Prevalence and sociodemographic associations of common mental disorders in a nationally representative sample of the general population of Greece. BMC psychiatry. [Internet]. 2013 [acesso em 07 jan 2019]; 13(163). Disponível em: http://dx.doi.org/10.1186/1471-244X-13-163.

4. Santos MM dos, Nascimento FF do, Cabral S de MR, Oliveira ES de, Santos RM dos, Carvalho L de S. Relação bilateral entre excesso de peso e transtornos mentais. Rev Bras Promoç Saúde [Internet]. 2018 [acesso em 15 abr 2020]; 31(1). Disponível em: http://dx.doi.org/10.5020/18061230.2018.6740. 
6. Kalarchian, MA, King WC, Devlin MJ, Marcus MD, Yanovski SZ, Mitchell JE, et al. Mental disorders and weight change in a prospective study of bariatric surgery patients: 7 years of follow-up. Surg Obes Relat Dis [Internet]. 2019 [acesso em 15 abr 2020]; 15(5). Disponível em: https://doi.org/10.1016/j. soard.2019.01.008.

7. Dawes AJ, Maggard-Gibbons M, Maher AR, Booth MJ, Miake-Lye BAi, Beores JM, et al. Mental health conditions among patients seeking and undergoing bariatric surgery: a meta-analysis. JAMA. [Internet]. 2016 [acesso em 07 jan 2019]; 315(2). Disponível em: http://dx.doi.org/10.1001/jama.2015.18118.

8. Luppino FS, Wit LM de, Bouvy PF, Stijnen T, Cuijpers P, Penninx BWJH, et al. Overweight, obesity and depression: a systematic meta-analysis of longitudinal studies. Arch Gen Psychiatry. [Internet]. 2010 [acesso em 15 abr 2020]; 67(3). Disponível em: http://dx.doi.org/10.1001/archgenpsychiatry.2010.2.

9. Gregorio VD, Lucchese R, Vera I, Silva GC, Silva A, Moraes RCC. The alcohol consumption is amended after bariatric surgery? An integrative review. ABCD, arq. bras. cir. dig. [Internet]. 2016 [acesso em 07 jan 2019]; 29(suppl.1). Disponível em: http://dx.doi.org/10.1590/0102-6720201600S10027.

10. Gregorio VD, Ramalho MF, Santiago JB, Lucchese R, Vera I, Lemos MF, et al. Fatores associados ao tabagismo em pacientes pós-cirurgia bariátrica. Rev Bras Promoç Saúde [Internet]. 2018 [acesso em 15 abr 2020]; 31(1). Disponível em: http://dx.doi.org/10.5020/18061230.2018.7112.

11. Lage-Hansen PR, Holm J, Gram J, Larsen K. Sleep apnoea in patients undergoing bariatric surgery. Dan. Med. J. [Internet] 2018 [acesso em 15 abr 2020]; 65(2). Disponível em: https://www.ncbi.nlm.nih.gov/ pubmed/29393036.

12. Nagendran M, Carlin AM, Bacal D, Genaw JA, Hawasli AA, Birkmeyer NJ, et al. Self-reported remission of obstructive sleep apnea following bariatric surgery: cohort study. Surg. obes. relat. dis. [Internet]. 2015 [acesso em 07 jan 2019]; 11(3). Disponível em: http://dx.doi.org/10.1016/j. soard.2014.10.011.

13. Instituto de Estudo de Saúde Suplementar (IEES), org. Cirurgia bariátrica. Pareceres técnicoscientíficos [Internet]. Rio de Janeiro: IESS; 2012 [acesso em 04 abr 2019]. Volume 1. Disponível em: https://pt.scribd.com/document/220309638/phpiuO8fP\#fullscreen\&from embed.

14. Saules KK, Wiedemann A, Ivezaj V, Hopper JA, Foster-Hartsfield J, Schwarz D. Bariatric surgery history among substance abuse treatment patients: prevalence and associated features. Surg. obes. relat. dis. [Internet]. 2010 [acesso em 07 jan 2019]; 6(6). Disponível em: http://dx.doi.org/10.1016/j. soard.2009.12.008.

15. Gonçalves DA, Mari J de J, Bower P, Gask L, Dowrick C, Tófoli LF, et al. Brazilian multicentre study of common mental disorders in primary care: rates and related social and demographic factors. Cad. Saúde Pública. [Internet]. 2014 [acesso em 07 jan 2019]; 30(3). Disponível em: https://doi.org/10.1590/0102311X00158412.

16. Lima MG, Barros MB de A, Alves MCGP. Sleep duration and health status self-assessment (SF-36) in the elderly: a population-based study (ISA-Camp 2008). Cad. Saúde Pública. [Internet]. 2012 [acesso em 07 jan 2019]; 28(9). Disponível em: http://dx.doi.org/10.1590/S0102-311X2012000900007.

17. Silva TM da, Aguiar OB de, Fonseca M de JM da. Associação entre sobrepeso, obesidade e transtornos mentais comuns em nutricionistas. J. bras. psiquiatr. [Internet]. 2015 [acesso em 07 jan 2019]; 64(1). Disponível em: http://dx.doi.org/10.1590/0047-2085000000053.

18. Machado CE, Zilberstein B, Cecconello I, Monteiro M. Compulsão alimentar antes e após a cirurgia bariátrica. ABCD, arq. bras. cir. dig. [Internet]. 2008 [acesso em 07 jan 2019]; 21(4). Disponível em: https:// doi.org/10.1590/S0102-67202008000400007.

19. Lent MR and Swencionis C. Addictive personality and maladaptive eating behaviors in adults seeking bariatric surgery. Eat. behav. [Internet]. 2012 [acesso em 07 jan 2019]; 13(1). Disponível em: http://dx.doi. org/10.1016/j.eatbeh.2011.10.006. 
20. Cronlein T. Insomnia and obesity. Curr Opin Psychiatry. [Internet]. 2016 [acesso em 07 jan 2019]; 29(6):409-12. Disponível em: http://dx.doi.org/10.1097/YCO.0000000000000284.

21. Qian J, Morris CJ, Caputo R, Garaulet M, Scheer FAJL. Ghrelin is impacted by the endogenous circadian system and by circadian misalignment in humans. Int. J. obes. [Internet]. 2018 [acesso em 07 jan 2019]; 43. Disponível em: http://dx.doi.org/10.1038/s41366-018-0208-9.

22. Meany G, Conceição E, Mitchell JE. Binge eating, binge eating disorder and loss of control eating: effects on weight outcomes after bariatric surgery. Eur. Eat. Disord. Rev [Internet]. 2014 [acesso em 15 abr 2020]; 22(2). Disponível em: http://dx.doi.org/10.1002/erv.2273.

23. Lluch A, Herbeth B, Méjean L, Siest G. Dietary intakes, eating style and overweight in the Stanislas Family Study. Int J Obes Relat Metab Disord [Internet] 2000 [acesso em 15 abr 2020]; 24(11). Disponível em: https://www.nature.com/articles/0801425.

24. Small L, Aplasca A. Child Obesity and Mental Health: a complex interaction. Child Adolesc Psychiatr Clin N Am. [Internet]. 2016 [acesso em 07 jan 2019]; 25(2). Disponível em: https://doi.org/10.1016/j. chc.2015.11.008.

25. Adams RE, Bukowski WM. Peer victimization as a predictor of depression and body mass index in obese and non-obese adolescents. J Child Psychol Psychiatry [Internet] 2008 [acesso em 15 abr 2020]; 49(8). Disponível em: https://doi.org/10.1111/j.1469-7610.2008.01886.x.

26. Eisenberg ME, Neumark-Sztainer D, Story M. Associations of weight-based teasing and emotional well-being among adolescents. Arch Pediatr Adolesc Med [Internet]. 2003 [acesso em 15 abr 2020]; 157(8). Disponível em: http://doi.org/10.1001/archpedi.157.8.733.

27. Russell-Mayhew S, McVey G, Bardick A, Ireland A. Mental health, wellness, and childhood overweight/ obesity. J obes. [Internet]. 2012 [acesso em 07 jan 2019]; (ID281801). Disponível em: https://doi. org/10.1155/2012/281801.

28. Wee CC, Mukamal KJ, Huskey KW, Apovian CM, Jones DBM, Blackburn GL, et al. High-risk alcohol use after weight loss surgery. Surg Obes Relat Dis [Internet]. 2014 [acesso em 15 abr 2020]; 10(3). Disponível em: http://doi.org/10.1016/j.soard.2013.12.014.

29. Conason A, Teixeira J, Hsu C-H, Puma L, Knafo D, Geliebter A. Substance use following bariatric weight loss surgery. JAMA surgery [Internet]. 2013 [acesso em 15 abr 2020]; 148(2). Disponível em: http:// doi.org/10.1001/2013.jamasurg.265.

30. Gabert DL, Majumdar SR, Sharma AM, Rueda-Clausen CF, Klarenbach SW, Birch DW, et al. Prevalence and predictors of self-reported sexual abuse in severely obese patients in a population-based bariatric program. J Obes. [Internet]. 2013 [acesso em 07 jan 2019]; (ID374050). Disponível em: http://dx.doi. org/10.1155/2013/374050. 
Recebido: $13 / 05 / 2019$

Finalizado: 26/05/2020

Editora associada: Susanne Elero Betiolli

Autor Correspondente:

Graciele Cristina Silva

Universidade Federal de Goiás

Av. Dr. Lamartine Pinto de Avelar, 1120 - 75704-020 - Catalão, GO, Brasil

E-mail: gcsilvanut@gmail.com

Contribuição dos autores:

Contribuições substanciais para a concepção ou desenho do estudo; ou a aquisição, análise ou interpretação de dados do estudo - SBE, LMSC, VDG, GCS

Elaboração e revisão crítica do conteúdo intelectual do estudo - RL, IV, MFL

Aprovação da versão final do estudo a ser publicado - MFL

Responsável por todos os aspectos do estudo, assegurando as questões de precisão ou integridade de qualquer parte do estudo - VDG

cc) (1)

Este obra está licenciado com uma Licença Creative Commons Atribuição 4.0 Internacional. 